\title{
Optimal Vaccine Distribution Strategy for Different Age Groups of Population: A Differential Evolution Algorithm Approach
}

\author{
Xiao-Min Hu, ${ }^{1}$ Jun Zhang, ${ }^{2}$ and Haihong Chen ${ }^{3}$ \\ ${ }^{1}$ Guangdong Key Laboratory of Health Informatics, Health Information Research Center, School of Public Health, \\ Sun Yat-sen University, Guangzhou 510080, China \\ ${ }^{2}$ Key Laboratory of Machine Intelligence and Advanced Computing of Ministry of Education of China, Engineering Research Center \\ of Supercomputing Engineering Software of Ministry of Education of China, School of Advanced Computing, Sun Yat-sen University, \\ Guangzhou 510006, China \\ ${ }^{3}$ Department of Respiratory Medicine, First Affiliated Hospital of Sun Yat-sen University, Guangzhou 510080, China
}

Correspondence should be addressed to Xiao-Min Hu; xmhu@ieee.org

Received 19 May 2014; Accepted 29 July 2014; Published 27 August 2014

Academic Editor: Hailin Liu

Copyright (C) 2014 Xiao-Min Hu et al. This is an open access article distributed under the Creative Commons Attribution License, which permits unrestricted use, distribution, and reproduction in any medium, provided the original work is properly cited.

\begin{abstract}
Vaccination is one of the effective ways for protecting susceptible individuals from infectious diseases. Different age groups of population have different vulnerability to the disease and different contact frequencies. In order to achieve the maximum effects, the distribution of vaccine doses to the groups of individuals needs to be optimized. In this paper, a differential evolution (DE) algorithm is proposed to address the problem. The performance of the proposed algorithm has been tested by a classical infectious disease transmission model and a series of simulations have been made. The results show that the proposed algorithm can always obtain the best vaccine distribution strategy which can minimize the number of infectious individuals during the epidemic outbreak. Furthermore, the effects of vaccination on different days and the vaccine coverage percentages have also been discussed.
\end{abstract}

\section{Introduction}

Infectious diseases are harmful to public health and have made millions of economic loses each year [1]. The most common ways for preventing the spread of infectious diseases are isolation and vaccination. Isolation makes a physical block between infectious individuals and healthy individuals by reducing their direct contacts. However, pathogenic viruses may still spread through the air. Vaccination is a more effective method by developing adaptive immunity of a person to a pathogen $[2,3]$. The period of immunity may cover the whole epidemic of the disease or even a whole life. However, the production of vaccines needs time and money. In order to make the best meet between the cost and effect, an optimal vaccine distribution scheme is highly demanded.

The general way for distributing vaccines is to protect the individuals who are most likely exposed to the virus (e.g., doctors and nurses) and those who are the most vulnerable (e.g., children and the elderly) $[4,5]$. The transmission of an epidemic disease follows a rule. First, susceptible individuals will be exposed to a disease. Some of them will be infected by direct or indirect contacts. The infected individuals may recover themselves or be treated in hospital. The propagation scope and development of the disease can be recorded and analyzed. If we can estimate the infectious feature of the disease and the activities of the individuals, a better vaccine distribution scheme can be designed. Mossong et al. [6] pointed out that social contacts between different groups are relevant to the spread of infectious disease. Individuals in different age groups have different vulnerability to a disease [7]. Therefore, a vaccine distribution strategy is proposed in this paper for different age groups of individuals.

The objective of the optimization problem is to minimize the total infectious population since the outbreak of the disease until the epidemic seizes. In the literature, vaccine distribution mainly focuses on the transmissibility and infection risk of the disease and the vulnerability of individuals [810]. Longini Jr. and Halloran [11] proposed a strategy for the 


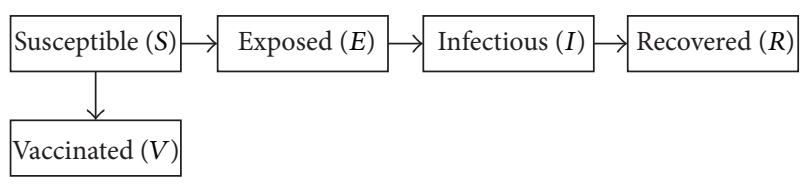

FIGURE 1: Schematic illustration of the transmission of an influenza pandemic.

distribution of influenza vaccines to high-risk groups. Patel et al. [12] proposed a genetic algorithm to find the optimal vaccine distributions to minimize the number of illnesses or deaths in the population. Tuite and his partners [13] used a transmission model to explain spatial spread of disease and identify optimal control interventions.

For solving the vaccine distribution optimization problem, we propose the use the differential evolution (DE) algorithm $[14,15]$ to enhance the effectiveness of vaccine protection. DE is a type of evolutionary algorithms [16-23] which are inspired by the natural evolution of the survival of the fittest. It is very suitable for solving continuous optimization problems and has achieved promising performance in searching nonlinear and multimodal space [24]. In this paper, a DE algorithm for optimizing the vaccine distribution strategy is proposed and a series of simulations are made for analyzing the results and the performance of the algorithm.

The rest of the paper is constructed as follows. Section 2 introduces the infectious disease transmission and control models in the literature. Section 3 describes the implementation of the proposed algorithm. Simulations and analysis are conducted in Section 4. Conclusions are made in Section 5.

\section{Infectious Disease Transmission and Control}

The risk of an infectious disease is reflected by a series of measures, such as the number of deaths, infections, and economic costs. Researchers have made different models and simulations to reflect the transmission and development of an epidemic disease. The most notable models are the SIR, SIS, and SIRS and their variations SEIS, SEIR, MSIR, MSEIR, and MSEIRS models ( $S$ : susceptible, $I$ : infected, $R$ : removed, $E$ : exposed in the latent period, and $M$ : passive immune) [25]. As an example, the transmission of an epidemic disease by vaccination treatments can be illustrated in Figure 1 by an influenza pandemic. The example is an SEIR model with the vaccination control method.

In Figure 1, suppose that a fixed number of individuals in a population are initially susceptible to the disease (the compartment $S$ ). Later, some of the susceptible individuals will be vaccinated and supposed to be immune to the disease (the compartment $V$ ) immediately. The others will be exposed to the disease in the latent period (the compartment E). The exposed individuals may be infected (the compartment $I$ ). The infected individuals may recover (the compartment $R$ ) and, in this model, they are supposed to be immune to the disease during the single wave of the disease.
The transmission and control of the above example can also be described by the following nonlinear differential equations according to [26]:

$$
\begin{gathered}
\dot{S}_{i}(t)=-\lambda_{i}(t)\left(S_{i}(t)-v_{i}(t)\right)-v_{i}(t), \\
\dot{E}_{i}(t)=-\tau_{i} E_{i}(t)+\lambda_{i}(t)\left(S_{i}(t)-v_{i}(t)\right), \\
\dot{I}_{i}(t)=-\gamma_{i} I_{i}(t)+\tau_{i} E_{i}(t), \\
\dot{R}_{i}(t)=\gamma_{i} I_{i}(t),
\end{gathered}
$$

where $S_{i}(t), E_{i}(t), I_{i}(t)$, and $R_{i}(t)$ are the numbers of individuals in the corresponding compartment, respectively. The differential equations of $\dot{S}_{i}(t), \dot{E}_{i}(t), \dot{I}_{i}(t)$, and $\dot{R}_{i}(t)$ represent the variations of the number of individuals in the corresponding compartments. The variable $v_{i}(t)$ is the number of individuals which are vaccinated at each time $t, \lambda_{i}(t)$ denotes the risk of virus infection of the suspected individuals during the period of latency, and $\tau_{i}$ is the probability of the exposed individuals to be infected by the disease. The infected individuals will recover with the rate $\gamma_{i}$. Different groups of individuals have different values of the rates because of their ages, occupations, or community, and so forth. The subscript $i$ is thus used to represent the different groups of individuals.

The infection risk $\lambda_{i}(t)$ for group $i$ relates to the virus transmission variable which relates to the proportion of susceptible individuals in the population $S_{i}(t) / P_{i}$, the vulnerability of the population $\beta_{i}$, the average number of contacts $c_{i j}$ between different groups $i$ and $j$ in the population, the number of infectious individuals $I_{j}(t)$, and the number of individuals $P_{j}$ in group $j$ [26]. The value of $\lambda_{i}$ at time $t$ can be computed by

$$
\lambda_{i}(t)=\frac{1}{n} \beta_{i} \frac{S_{i}(t)}{P_{i}} \sum_{j=1}^{n} c_{i j} \frac{I_{j}(t)}{P_{j}},
$$

where $n$ is the number of the predefined groups.

Vaccination is a way which can reduce the number of susceptible individuals under the risk of the disease by stimulating an individual's immune system to protect them. Therefore, the infection of the disease can be suppressed to a smaller group of susceptible individuals by vaccination. Because the production of vaccines is usually expensive and needs time, in order to achieve the best results, the distribution of vaccines to the susceptible individuals needs to be optimized. In this paper, the vaccines are supposed to be applied to the candidate individuals on a predefined day $t_{\text {vacc }}$ during the course of the epidemic wave. Suppose that the duration of a single epidemic wave is $T$. So we have $0<t_{\text {vacc }}<$ $T$. Therefore, the objective function can be represented by

$$
\text { Minimize } f\left(V\left(t_{\text {vacc }}\right)\right)=\int_{t=t_{\text {vacc }}}^{T} \sum_{i=1}^{n} I_{i}(t) d t,
$$

where $V\left(t_{\text {vacc }}\right)=\left(v_{1}\left(t_{\text {vacc }}\right), v_{2}\left(t_{\text {vacc }}\right), \ldots, v_{n}\left(t_{\text {vacc }}\right)\right)$ represents a vaccine distribution scheme at time $t_{\text {vacc }}$ for each group. The objective for controlling infectious diseases by vaccination is to find the optimal vaccine distribution scheme which can 
minimize the total number of infectious individuals during the course of the epidemic wave if the vaccination is applied on the day $t_{\text {vacc }}$.

In the considered problem, the number of individuals in the corresponding compartment is updated each day. Therefore, the above integral is actually calculated as

$$
f\left(V\left(t_{\mathrm{vacc}}\right)\right)=\sum_{t=t_{\mathrm{vacc}}}^{T} \sum_{i=1}^{n} I_{i}(t) .
$$

In this paper, the DE algorithm is used to address the problem.

\section{Implementation of the Proposed Algorithm}

In this section, the implementation of the DE algorithm for the vaccine distribution will be presented. The optimization process of the DE algorithm includes initialization, mutation, crossover, and selection operations.

3.1. Initialization. The search process of the DE algorithm is based on the evolution of a group of individuals, which are the indirect representation of the candidate solutions of the problem and are encoded according to the optimization objective. Note that the term individual in the DE algorithm represents its basic optimization unit and it is different from the individual in the SEIR model. In the vaccine distribution problem, the vaccine distribution must satisfy

$$
\sum_{i=1}^{n} v_{i}\left(t_{\mathrm{vacc}}\right) \leq V_{\max }\left(t_{\mathrm{vacc}}\right)
$$

where $V_{\max }\left(t_{\text {vacc }}\right)$ is the total number of vaccine doses which can be applied at time $t_{\text {vacc }}$. It is known that the more vaccines are applied, the better they are for controlling the epidemic, so the total amount of vaccines at time $t_{\text {vacc }}$ is considered in this paper.

Each individual $k$ in the DE algorithm for the $G$ th generation is encoded as $X_{k}^{(G)}=\left\{x_{k, 1}^{(G)}, x_{k, 2}^{(G)}, \ldots, x_{k, n-1}^{(G)}\right\}$, representing the percentage of the remaining vaccine doses which will be used for the $i$ th group of individuals, $k=$ $1,2, \ldots, m$, and $m$ is the number of individuals used in the algorithm. Note that we have $x_{k, i}^{(G)} \in[0,1], i=1,2, \ldots, n-1$. Therefore, the vaccine distribution strategy for the $n$ groups of individuals based on the encoded individual $k$ is

$$
\begin{gathered}
v_{1}=V_{\max } x_{k, 1}^{(G)}, \\
v_{i}=\left(V_{\max }-\sum_{j=1}^{i-1} v_{j}\right) x_{k, i}^{(G)}, \quad i=2,3, \ldots, n-1, \\
v_{n}=V_{\max }-\sum_{j=1}^{n-1} v_{j} .
\end{gathered}
$$

In the above equations, the variable $t_{\text {vacc }}$ is omitted for better clarification. The $m$ individuals in the initial population of the algorithm are randomly generated.
3.2. Mutation and Crossover. After initialization, the mutation operation will be used to produce a mutant vector $V_{k}^{(G)}=$ $\left(v_{k, 1}^{(G)}, v_{k, 2}^{(G)}, \ldots, v_{k, n-1}^{(G)}\right)$ with respect to each individual $X_{k}^{(G)}$ in the current population in the $G$ th generation, $k=1,2, \ldots, m$. $V_{k}^{(G)}$ is generated via a mutation strategy. In this paper, $V_{k}^{(G)}$ is computed by

$$
V_{k}^{(G)}=X_{\text {best }}^{(G)}+F \cdot\left(X_{r_{1}}^{(G)}-X_{r_{2}}^{(G)}\right) .
$$

The indices $r_{1}$ and $r_{2}$ are different uniform random integer numbers generated in the range $[1, m]$ and $X_{\text {best }}^{(G)}$ is the recorded best individual that is found by the algorithm. The scaling factor $F$ is a positive predefined parameter for scaling the different vector.

After the mutation operation, each pair of the vector $X_{k}^{(G)}$ and its corresponding mutant vector $V_{k}^{(G)}$ will be used to generate a trial vector $U_{k}^{(G)}=\left(u_{k, 1}^{(G)}, u_{k, 2}^{(G)}, \ldots, u_{k, n-1}^{(G)}\right)$, and the process is called crossover. The crossover operation is defined as

$$
u_{k, i}^{(G)}= \begin{cases}v_{k, i}^{(G)}, & \text { if } \operatorname{rand}(0,1) \leq C R \text { or } i=i_{\text {rand }}, \\ x_{k, i}^{(G)}, & \text { otherwise, }\end{cases}
$$

where rand $(0,1)$ is a uniform random value in the range $(0,1)$ and $i_{\text {rand }}$ is a random integer number chosen from 1 to $n-1$ for each individual $k, k=1,2, \ldots, m, i=1,2, \ldots, n-1$. The crossover rate $C R$ is a predefined parameter in the range $[0,1)$.

3.3. Selection. After mutation and crossover, the newly generated individuals $U_{1}^{(G)}, U_{2}^{(G)}, \ldots, U_{m}^{(G)}$ will be evaluated and the selection operation is performed. The selection operation is defined by

$$
X_{k}^{(G+1)}= \begin{cases}U_{k}^{(G)}, & \text { if } f\left(V_{U_{k}^{(G)}}\right) \leq f\left(V_{X_{k}^{(G)}}\right) \\ X_{k}^{(G)}, & \text { otherwise. }\end{cases}
$$

DE compares the objective function value of the vaccine distribution strategy by each trial vector $U_{k}^{(G)}$ and its corresponding vector $X_{k}^{(G)}$ in the current population. Since the objective of the problem is to minimize the total number of the infectious population, if the objective function value of the trial vector $U_{k}^{(G)}$ satisfies $f\left(V_{U_{k}^{(G)}}\right) \leq f\left(V_{X_{k}^{(G)}}\right)$, the vector $X_{k}^{(G)}$ will be replaced by the trial vector and will join in the population of the next generation. Otherwise, the vector $X_{k}^{(G)}$ will be reserved in the next generation.

The flowchart of the DE algorithm is illustrated in Figure 2. Through a series of generations of the mutation, crossover, evaluation, and selection operations, the best individual is updated and the algorithm stops when the termination condition is satisfied (e.g., the algorithm reaches the predefined maximum generation number).

\section{Simulations and Analysis}

In the experimental section, the performance of the proposed DE algorithm will be compared with some vaccine deployment strategies in the literature. First, the settings of the simulations are described. 


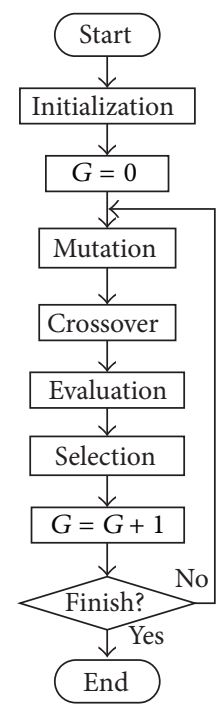

FIgURE 2: Flowchart of the DE algorithm.

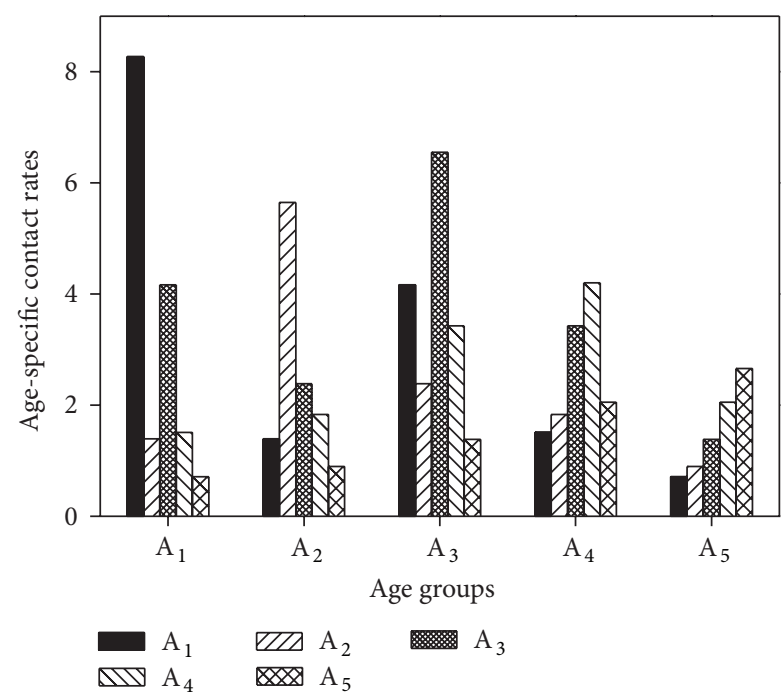

FIGURE 3: Illustration of the contact rates between age groups.

4.1. Settings of the Simulations. The epidemic model in [26] will be used in the simulation. The $n=5$ age groups of population considered in the experiment are $A_{1}$ (5-14 years), $A_{2}$ (15-24 years), $A_{3}$ (25-44 years), $A_{4}$ (45-64 years), and $A_{5}$ (65 or above years). In the pandemic transmission model, the vulnerability values of the population are $\beta_{1}=0.434$, $\beta_{2}=0.158, \beta_{3}=0.118, \beta_{4}=0.046$, and $\beta_{5}=0.046$. The probabilities of the exposed individuals to be infected by the disease and the recovery rates are $\tau_{i}=0.25, \gamma_{i}=0.334$, $i=1,2, \ldots, 5$, the same for the five age groups.

The numbers of contacts between different age groups are illustrated in Figure 3. It can be observed that the individuals in the same group contact more frequently than those in the other groups. Youngsters in group $A_{1}$ have the highest contact frequency and the individuals in $A_{3}$ are the next.

The initial points of the infection of the disease affect the epidemic spreading. The vaccine coverage and releasing time also influence the total number of infectious population. For the purpose of evaluation, in the simulation, initially 30 individuals in $A_{2}$ are set exposed to the disease. The composition of the population in each age group is $A_{1}=$ $0.94 \times 10^{6}, A_{2}=0.91 \times 10^{6}, A_{3}=2.30 \times 10^{6}, A_{4}=1.86 \times 10^{6}$, and $A_{5}=0.85 \times 10^{6}$. Various vaccine coverage and the releasing time will be simulated in the experiment and the time range $T=300$. The parameters in the $\mathrm{DE}$ algorithm are set as the population size is $m=20, F=0.5$, and $C R=0.4$. The maximum number of generations before the termination of the algorithm is 1000 .

4.2. Traditional Deployment Methods for Comparisons. In the literature, the vaccine deployment strategy is generally made according to the transmissibility of the disease, the vulnerability of the population, or the infection risk of the disease. In this paper, three common strategies (S1 to S3) are introduced.

Strategy 1 (S1): Based on Transmissibility. More vaccine doses will be given to the individuals with a higher contact frequency. The vaccination proportion $p_{i}$ for each age group $A_{i}$ at time $t_{\text {vacc }}$ is

$$
p_{i}(t)=\frac{\sum_{k=1}^{n} c_{i k}}{\sum_{j=1}^{n} \sum_{k=1}^{n} c_{j k}}, \quad i=1,2, \ldots, n .
$$

Strategy 2 (S2): Based on Vulnerability. This strategy focuses on the infectious vulnerability $\beta_{i}$. The number of vaccine doses for each population group is proportional to their infectious vulnerabilities. The vaccination proportion $p_{i}$ for each age group $A_{i}$ at time $t_{\text {vacc }}$ is

$$
p_{i}(t)=\frac{\beta_{i}}{\sum_{j=1}^{n} \beta_{j}}, \quad i=1,2, \ldots, n .
$$

Strategy 3 (S3): Based on Infection Risk. The vaccination proportion $p_{i}$ for each age group $A_{i}$ at time $t_{\text {vacc }}$ is based on the value of the time-dependent infection risk $\lambda_{i}(t)$; thus

$$
p_{i}(t)=\frac{\lambda_{i}(t)}{\sum_{j=1}^{n} \lambda_{i}(t)}, \quad i=1,2, \ldots, n .
$$

4.3. Comparisons with Different Deployment Methods. In the simulation, the vaccines are supposed to be released and applied to the individuals only once during one wave of the epidemic at time $t_{\text {vacc }}$ and the vaccines take effect immediately. Therefore, we first check the effectiveness of the vaccine deployment at different days $t_{\text {vacc }}=1,50,75$, and 100 . The vaccine coverage percentage, which is the total number of available vaccine doses, is set to be equal to $5 \%$ of the total population.

Figure 4 illustrates the curves of infectious population during the course of the epidemic. It can be observed that, without vaccination (no Vacc.), the infection dynamic curve of the epidemic is like a bell shape. If the vaccine deployment is applied, the peak of the infection dynamic curve will 


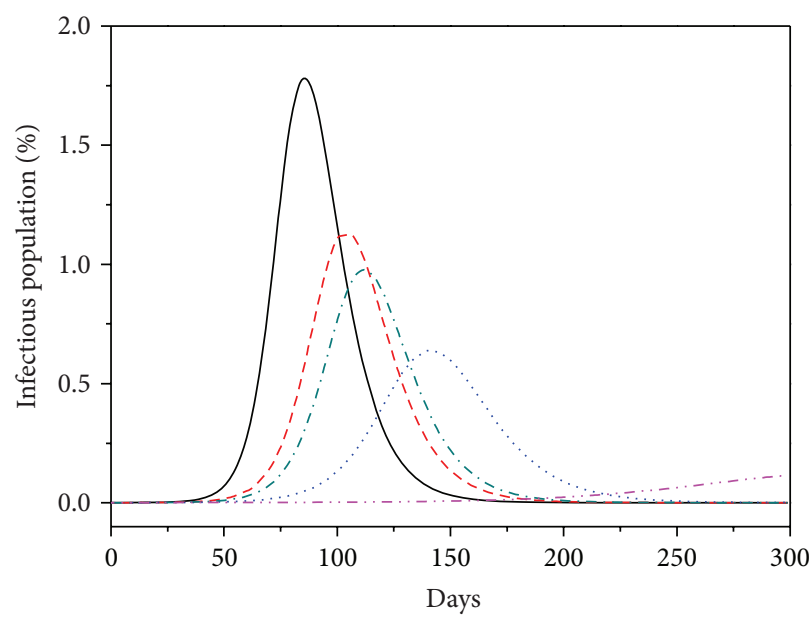

(a)

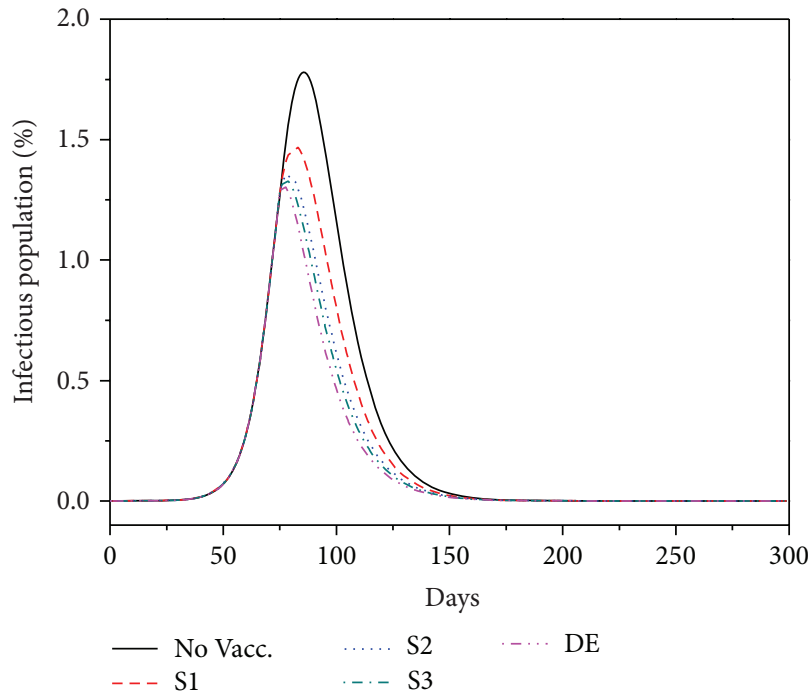

(c)

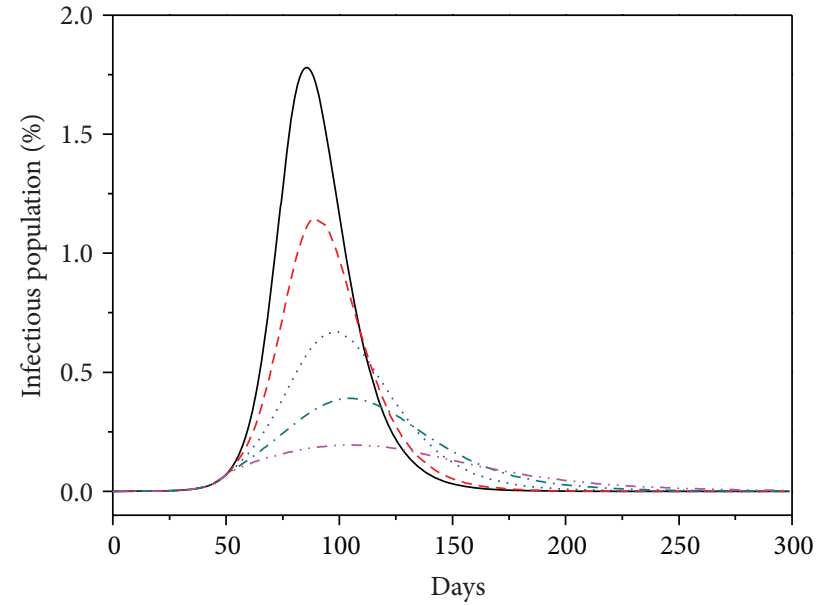

(b)

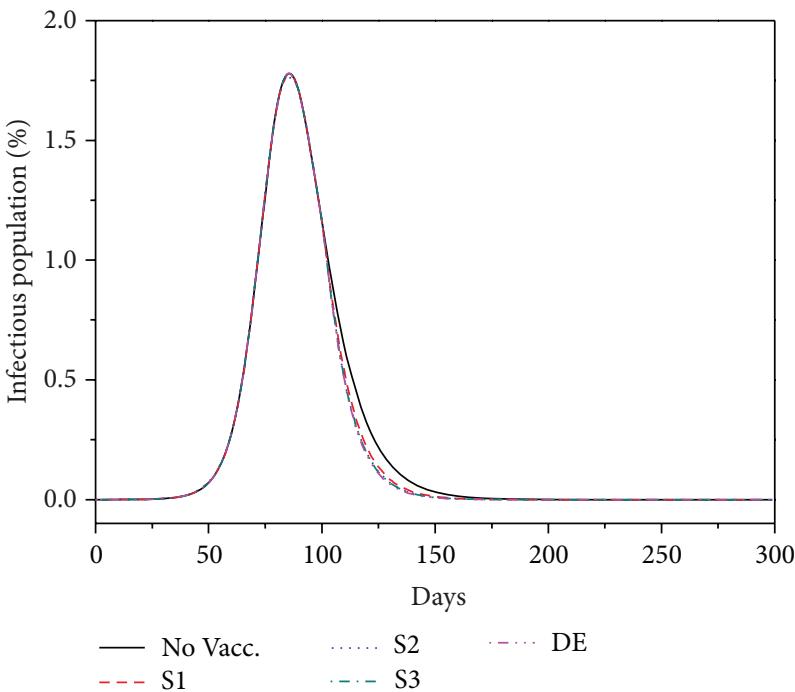

(d)

FIGURE 4: Infection dynamic curves for different methods with different vaccination days: (a) $t_{\text {vacc }}=1$, (b) $t_{\text {vacc }}=50$, (c) $t_{\text {vacc }}=75$, and (d) $t_{\text {vacc }}$ $=100$.

be reduced and delayed. Compared with the other three methods, the vaccine distribution strategy of DE is the best and its curve is the lowest. If the vaccines are applied on day $t_{\mathrm{vacc}}=1$, the best vaccine distribution by $\mathrm{DE}$ can mostly suppress the outbreak of the epidemic. If the vaccines are taken on day $t_{\mathrm{vacc}}=50$, the outbreak of the epidemic can be reduced to a small number of population. If the vaccines are applied later than the outbreak of the epidemic (e.g., day $t_{\mathrm{vacc}}$ $=100$ ), the effectiveness of vaccination will be unsatisfying to all methods.

Table 1 tabulates the summation of the number of infectious individuals each day after the vaccination is applied, that is, the objective function value for the different methods. It is shown that $\mathrm{DE}$ achieves the best result compared with the other three methods. By taking the vaccines as earlier, the total number of the infectious population is smaller. For example, if the vaccines are taken on day $t_{\mathrm{vacc}}=1$, the
TABLE 1: Comparisons of the summation of infectious individuals each day after the vaccination.

\begin{tabular}{lcccc}
\hline Methods & $t_{\text {vacc }}=1$ & $t_{\text {vacc }}=50$ & $t_{\text {vacc }}=75$ & $t_{\text {vacc }}=100$ \\
\hline DE & $\mathbf{5 . 1 4} \times 10^{5}$ & $\mathbf{1 . 5 1} \times 10^{6}$ & $\mathbf{2 . 1 3} \times 10^{6}$ & $\mathbf{9 . 2 2} \times 10^{5}$ \\
S1 & $2.82 \times 10^{6}$ & $3.61 \times 10^{6}$ & $3.03 \times 10^{6}$ & $1.00 \times 10^{6}$ \\
S2 & $3.64 \times 10^{6}$ & $2.82 \times 10^{6}$ & $2.54 \times 10^{6}$ & $9.42 \times 10^{5}$ \\
S3 & $3.33 \times 10^{6}$ & $2.19 \times 10^{6}$ & $2.35 \times 10^{6}$ & $9.40 \times 10^{5}$ \\
\hline
\end{tabular}

summation value is $5.14 \times 10^{5}$, almost one third of the value for the case with $t_{\text {vacc }}=50$.

4.4. Further Discussions. In the above experiment, the DE algorithm can always achieve the best vaccine distribution strategies. In the further experiment, the features of the vaccine distribution will be discussed. 


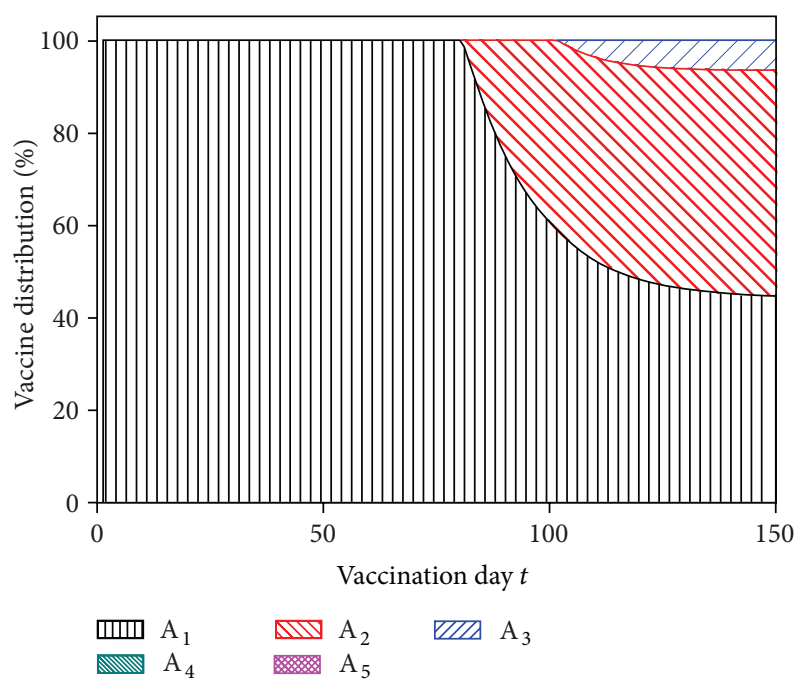

Figure 5: Area graph of the vaccine distribution for different age groups.

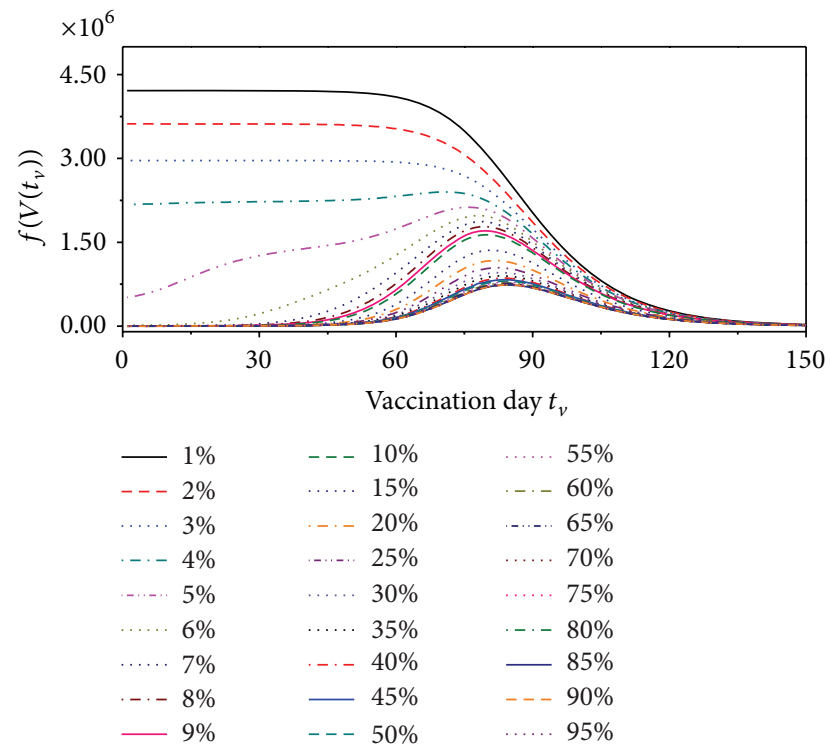

FIGURE 6: Objective function values for different vaccine coverage percentages and vaccination days.

The vaccine distribution by the DE algorithm for the five age groups is shown in Figure 5 for the cases with the vaccines taken on day $t_{\text {vacc }}=1$ to 150 and $5 \%$ vaccine coverage. It can be observed that more than $99.9 \%$ of the vaccines should be distributed to group $A_{1}$ if the vaccine doses can be available before the day $t_{\mathrm{vacc}}=80$. After that, the individuals in group $A_{2}$ will be the second focus of vaccination and, after day $t_{\text {vacc }}$ $=127$, the percentage of vaccine doses to $A_{2}$ surpasses that to $A_{1}$. After day $t_{\mathrm{vacc}}=104$, the doses for $A_{3}$ also increase obviously. The number of doses to $A_{4}$ and $A_{5}$ is too small to be seen in the figure.

Figure 6 shows the influence of different vaccine coverage percentages and vaccination days on the total number of infectious individuals on each day, which is the objective

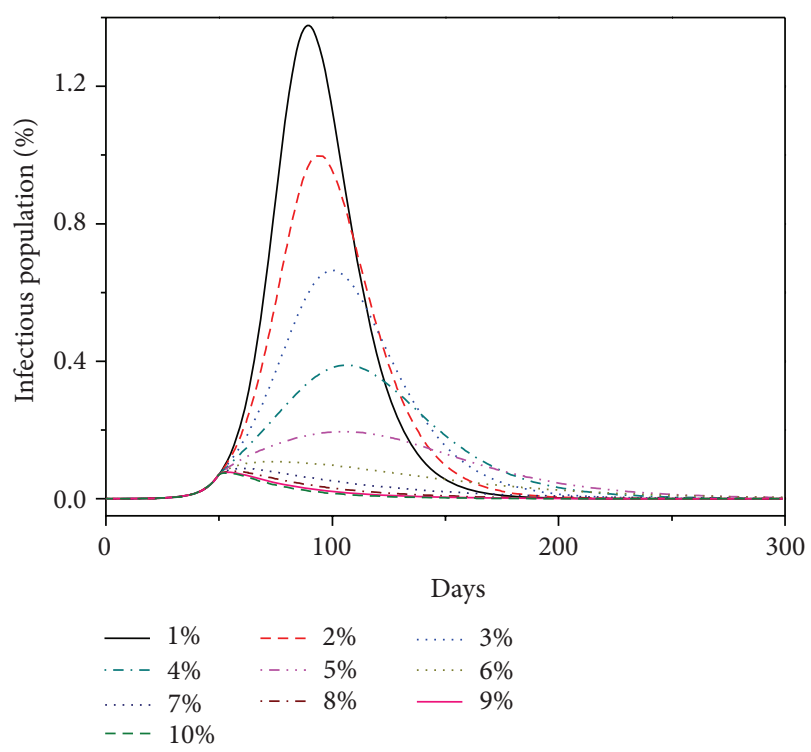

Figure 7: Infection dynamic curves for the vaccine coverage percentages $1 \%$ to $10 \%$ when vaccinated on day $t_{\text {vacc }}=50$.

function value of (3). It can be observed that the larger the vaccine coverage percentage, the smaller the number of infectious individuals with the same vaccination day. When the vaccine coverage percentage is from $1 \%$ to $10 \%$, the objective function value varies greatly. So we choose $t_{\text {vacc }}=$ 50 and compare the infection dynamic curves for the vaccine coverage percentages from $1 \%$ to $10 \%$ in Figure 7 . It is shown that if the vaccine coverage can increase to $7 \%$, the epidemic control by the vaccination strategy will be much better than the smaller coverage percentages. By considering the cost and effect, the $7 \%$ vaccine coverage percentage is preferred.

\section{Conclusion}

In this paper, a differential evolution (DE) algorithm is proposed to optimize the problem of vaccine distribution, the objective of which is to find the optimal distribution strategy to determine the number of vaccine doses to different age groups of population and minimize the infectious population during the pandemic. A series of simulations on an SEIR model have been used to test the performance of the DE algorithm. Although there are several strategies in the literature to guide the distribution of vaccine doses, the proposed DE algorithm can obtain the best results.

\section{Conflict of Interests}

The authors declare that there is no conflict of interests regarding the publication of this paper.

\section{Acknowledgments}

This work was supported in part by the National HighTechnology Research and Development Program (863 Program) of China no. 2013AA01A212, National Science Fund 
for Distinguished Young Scholars 61125205, National Natural Science Foundation of China under Grants 61202130 and 61332002, NSFC Joint Fund with Guangdong under Key Project U1201258 and U1135005, Guangdong Natural Science Foundation S2012040007948, Fundamental Research Funds for the Central Universities no. 12lgpy47, and Specialized Research Fund for the Doctoral Program of Higher Education 20120171120027.

\section{References}

[1] J. T. Wu, E. S. K. Ma, C. K. Lee et al., "The infection attack rate and severity of 2009 pandemic H1N1 influenza in Hong Kong," Clinical Infectious Diseases, vol. 51, no. 10, pp. 1184-1191, 2010.

[2] G. Chowell, C. Viboud, X. Wang, S. M. Bertozzi, and M. A. Miller, "Adaptive vaccination strategies to mitigate pandemic influenza: Mexico as a case study," PLoS ONE, vol. 4, no. 12, Article ID e8164, 2009.

[3] S. Lee, M. Golinski, and G. Chowell, "Modeling optimal agespecific vaccination strategies against pandemic influenza," Bulletin of Mathematical Biology, vol. 74, no. 4, pp. 958-980, 2012.

[4] T. Heikkinen and S. Heinonen, "Effectiveness and safety of influenza vaccination in children: European perspective," Vaccine, vol. 29, no. 43, pp. 7529-7534, 2011.

[5] J. T. Vietri, M. Li, A. P. Galvani, and G. B. Chapman, "Vaccinating to help ourselves and others," Medical Decision Making, vol. 32, no. 3, pp. 447-458, 2012.

[6] J. Mossong, N. Hens, M. Jit et al., "Social contacts and mixing patterns relevant to the spread of infectious diseases," PLoS Medicine, vol. 5, no. 3, article e74, 2008.

[7] A. L. Greer, A. Tuite, and D. N. Fisman, "Age, influenza pandemics and disease dynamics," Epidemiology and Infection, vol. 138, no. 11, pp. 1542-1549, 2010.

[8] J. Medlock and A. P. Galvani, "Optimizing influenza vaccine distribution," Science, vol. 325, no. 5948, pp. 1705-1708, 2009.

[9] M. J. Keeling and P. J. White, "Targeting vaccination against novel infections: risk, age and spatial structure for pandemic influenza in Great Britain," Journal of the Royal Society Interface, vol. 8, no. 58, pp. 661-670, 2011.

[10] Y. Yang, J. D. Sugimoto, M. Elizabeth Halloran et al., "The transmissibility and control of pandemic influenza a (H1N1) virus," Science, vol. 326, no. 5953, pp. 729-733, 2009.

[11] I. M. Longini Jr. and M. E. Halloran, "Strategy for distribution of influenza vaccine to high-risk groups and children," The American Journal of Epidemiology, vol. 161, no. 4, pp. 303-306, 2005.

[12] R. Patel, I. M. Longini Jr., and M. E. Halloran, "Finding optimal vaccination strategies for pandemic influenza using genetic algorithms," Journal of Theoretical Biology, vol. 234, no. 2, pp. 201-212, 2005.

[13] A. R. Tuite, J. Tien, M. Eisenberg, D. J. D. Earn, J. Ma, and D. N. Fisman, "Cholera epidemic in Haiti, 2010: using a transmission model to explain spatial spread of disease and identify optimal control interventions," Annals of Internal Medicine, vol. 154, no. 9, pp. 593-601, 2011.

[14] R. Storn and K. Price, "Differential evolution-a simple and efficient heuristic for global optimization over continuous spaces," Journal of Global Optimization, vol. 11, no. 4, pp. 341359, 1997.
[15] K. V. Price, R. M. Storn, and J. A. Lampinen, Differential Evolution-A Practical Approach to Global Approach to Global Optimization, Springer, Berlin, Germany, 2005.

[16] Y. Wang, Y. Jiao, and H. Li, "An evolutionary algorithm for solving nonlinear bilevel programming based on a new constraint-handling scheme," IEEE Transactions on Systems, Man and Cybernetics Part C, vol. 35, no. 2, pp. 221-232, 2005.

[17] Y. Wang and C. Dang, "An evolutionary algorithm for global optimization based on level-set evolution and latin squares," IEEE Transactions on Evolutionary Computation, vol. 11, no. 5, pp. 579-595, 2007.

[18] X. Hu and J. Zhang, "Minimum cost multicast routing using ant colony optimization algorithm," Mathematical Problems in Engineering, vol. 2013, Article ID 432686, 13 pages, 2013.

[19] X. Hu, J. Zhang, H. S. Chung, Y. Li, and O. Liu, "SamACO: variable sampling ant colony optimization algorithm for continuous optimization," IEEE Transactions on Systems, Man, and Cybernetics, Part B, vol. 40, no. 6, pp. 1555-1566, 2010.

[20] X. Hu, J. Zhang, Y. Yu et al., "Hybrid genetic algorithm using a forward encoding scheme for lifetime maximization of wireless sensor networks," IEEE Transactions on Evolutionary Computation, vol. 14, no. 5, pp. 766-781, 2010.

[21] X. Hu, J. Zhang, H. S. Chung, O. Liu, and J. Xiao, "An intelligent testing system embedded with an ant-colony-optimizationbased test composition method," IEEE Transactions on Systems, Man and Cybernetics Part C, vol. 39, no. 6, pp. 659-669, 2009.

[22] W. Chen, J. Zhang, Y. Lin et al., "Particle swarm optimization with an aging leader and challengers," IEEE Transactions on Evolutionary Computation, vol. 17, no. 2, pp. 241-258, 2013.

[23] W. Chen and J. Zhang, "Ant colony optimization for software project scheduling and staffing with an event-based scheduler," IEEE Transactions on Software Engineering, vol. 39, no. 1, pp. 117, 2013.

[24] A. K. Qin, V. L. Huang, and P. N. Suganthan, "Differential evolution algorithm with strategy adaptation for global numerical optimization," IEEE Transactions on Evolutionary Computation, vol. 13, no. 2, pp. 398-417, 2009.

[25] D. J. Daley and J. Gani, Epidemic Modeling: An Introduction, Cambridge University Press, 1999.

[26] J. Liu and S. Xia, "Toward effective vaccine deployment: a systematic study," Journal of Medical Systems, vol. 35, no. 5, pp. 1153-1164, 2011. 


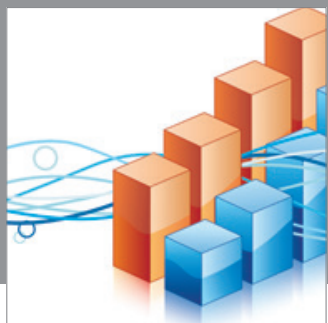

Advances in

Operations Research

mansans

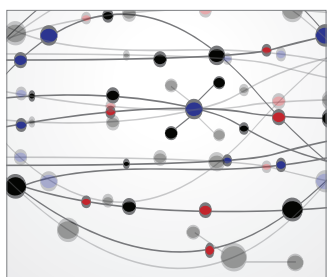

The Scientific World Journal
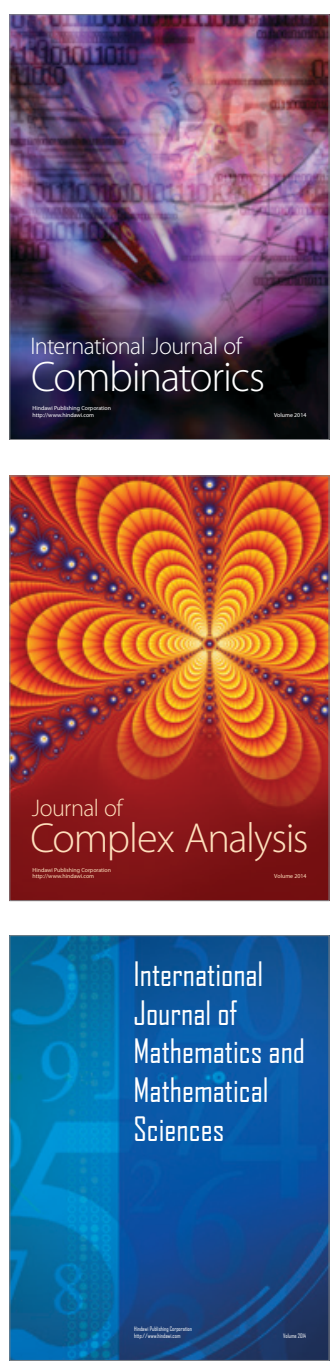
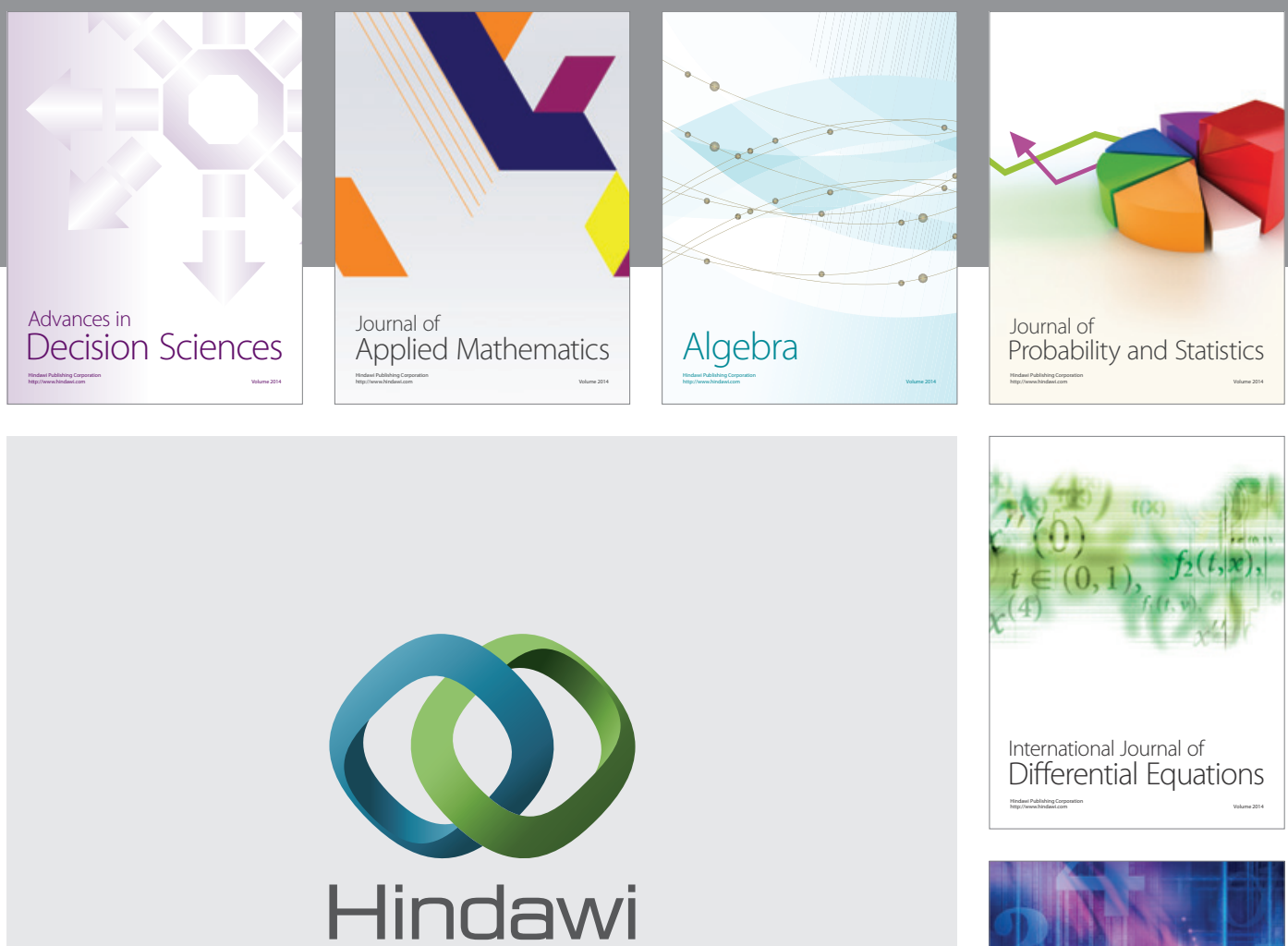

Submit your manuscripts at http://www.hindawi.com
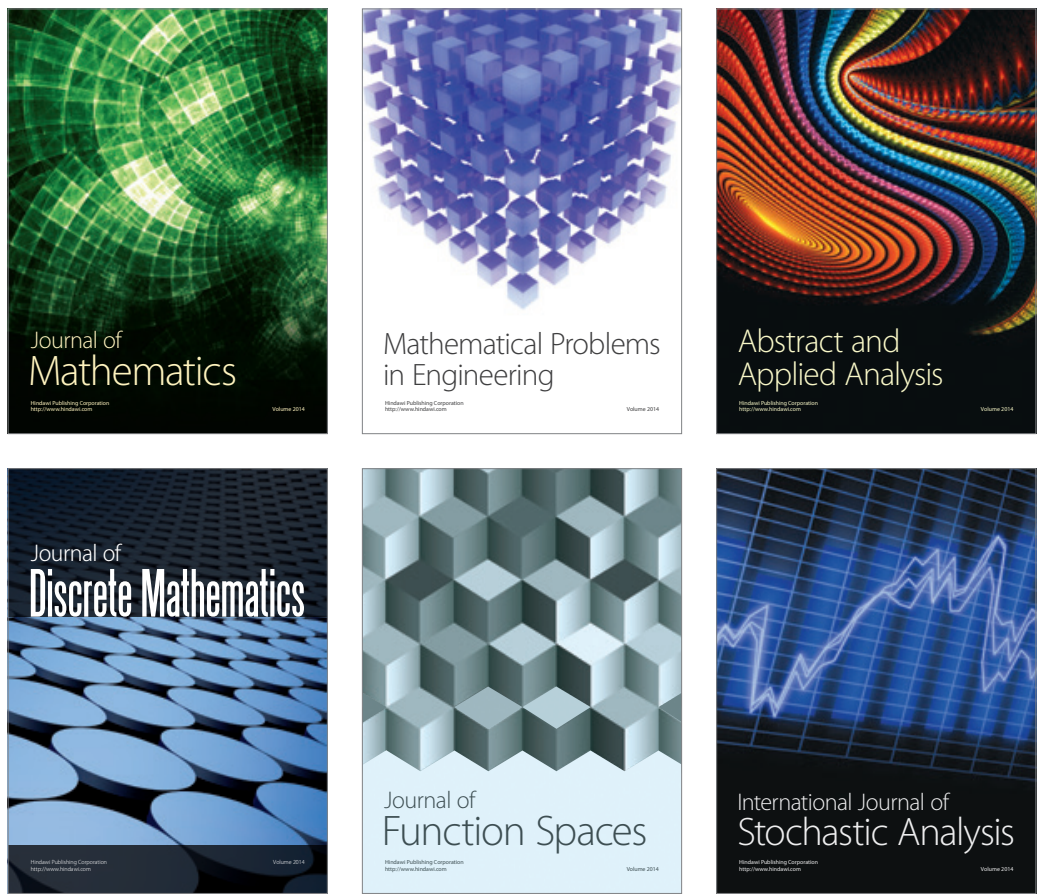

Journal of

Function Spaces

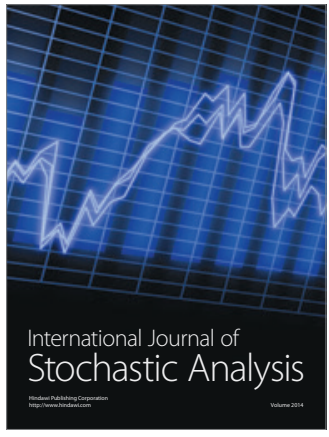

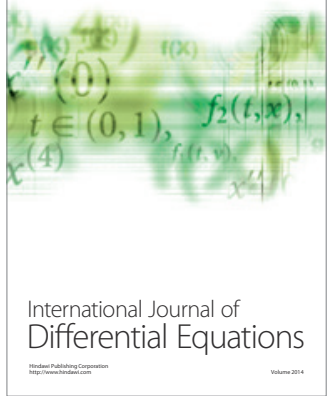
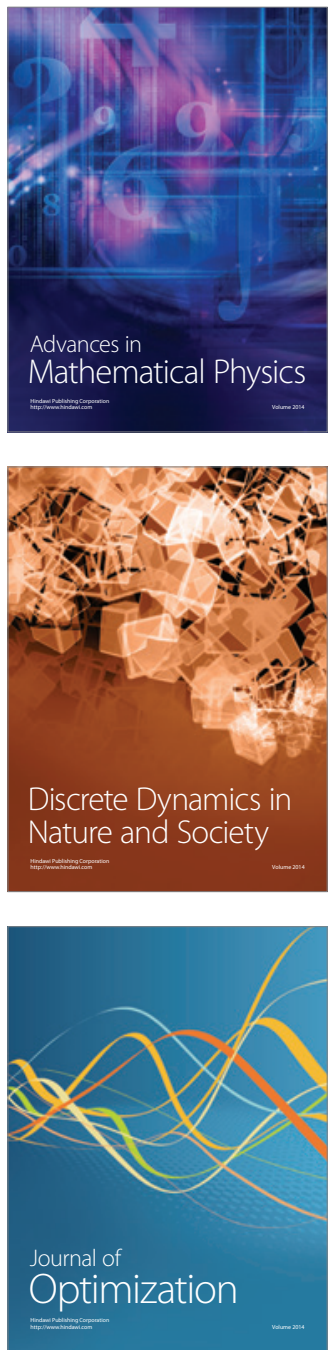